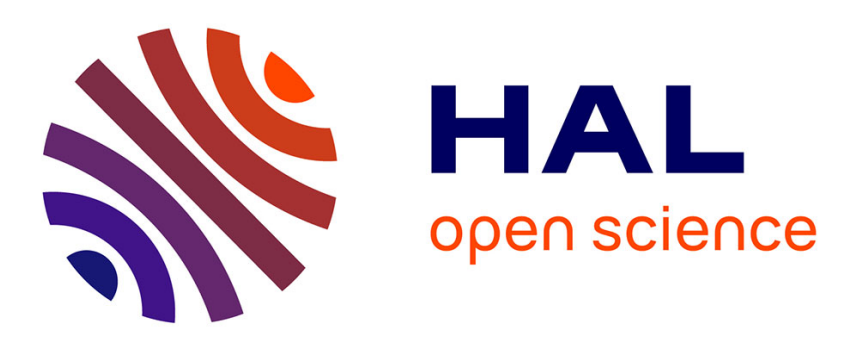

\title{
Squeezing in the many atom resonance fluorescence emitted in the forward direction: application to photon noise reduction
}

\author{
A. Heidmann, S. Reynaud
}

\section{- To cite this version:}

A. Heidmann, S. Reynaud. Squeezing in the many atom resonance fluorescence emitted in the forward direction: application to photon noise reduction. Journal de Physique, 1985, 46 (11), pp.1937-1948. 10.1051/jphys:0198500460110193700 . jpa-00210143

HAL Id: jpa-00210143

https://hal.science/jpa-00210143

Submitted on 1 Jan 1985

HAL is a multi-disciplinary open access archive for the deposit and dissemination of scientific research documents, whether they are published or not. The documents may come from teaching and research institutions in France or abroad, or from public or private research centers.
L'archive ouverte pluridisciplinaire HAL, est destinée au dépôt et à la diffusion de documents scientifiques de niveau recherche, publiés ou non, émanant des établissements d'enseignement et de recherche français ou étrangers, des laboratoires publics ou privés. 


\title{
Squeezing in the many atom resonance fluorescence emitted in the forward direction : application to photon noise reduction
}

\author{
A. Heidmann and S. Reynaud \\ Laboratoire de Spectroscopie Hertzienne de l'Ecole Normale Supérieure (*), 24, rue Lhomond, \\ F 75231 Paris Cedex 05, France
}

(Reçu le 24 mai 1985, accepté le 25 juin 1985)

\begin{abstract}
Résumé. - Nous montrons que les fluctuations du champ dans la fluorescence de résonance émise vers l'avant par un grand nombre d'atomes sont plus petites que celles du vide de rayonnement. Nous calculons, par un traitement quantique du champ et des atomes, cette « compression » des fluctuations et la réduction du bruit de photons qu'il est possible d'obtenir en entrant ce champ dans un détecteur homodyne ou hétérodyne. Cette étude théorique est restreinte à un milieu atomique mince et dilué, composé d'atomes à deux niveaux immobiles. Cependant nous étudions tous les cas d'excitation faible ou saturante, résonnante ou non résonnante et de détection homodyne ou hétérodyne. Nos résultats montrent qu'il est important de prendre en compte les propriétés de cohérence spatiotemporelle du champ pour évaluer correctement la réduction du bruit de photons.
\end{abstract}

\begin{abstract}
We show theoretically that the resonance fluorescence field radiated in the forward direction by an ensemble of many atoms exhibits squeezing. In a full quantum treatment we quantitatively evaluate this squeezing and the photon noise reduction which can be obtained by entering this squeezed field in a homodyne or heterodyne detector. The study is restricted to a dilute and thin atomic medium containing motionless two-level atoms but it includes all the cases of weak and saturating excitation, resonant and detuned excitation, homodyne and heterodyne detection. Our results show the importance of taking into account the field coherence properties for a correct evaluation of photon noise reduction.
\end{abstract}

\section{Introduction.}

Resonance fluorescence from a single two-level atom is known to reveal the non-classical properties of the electromagnetic field [1-4]. Some of them have been experimentally observed : photon antibunching [5-6], sub-Poissonian photon statistics [7], correlation between the photons emitted in the two sidebands of the fluorescence triplet [8]. The phenomenon of squeezing [9] has been shown to be present in single atom resonance fluorescence [10-14] but has not yet been observed.

The non-classical properties usually vanish when the field is radiated by many atoms. In this paper, we want to point out that it is not always true.

1.1 SQUEEZING IN THE FORWARD MANY ATOM RESONANCE FLUORESCENCE. - More precisely, we will show that the resonance fluorescence field radiated in the forward direction by an ensemble of many independent two-level atoms exhibits squeezing [15].

(*) Associé au C.N.R.S. (U.A 18).

JOURNAL DE PHYSIQUE. - T. 46, $\mathrm{N}^{\circ} 11$, NOVEMBRE 1985
The situation considered in this paper is sketched in figure 1. An atomic medium containing a great number of two-level atoms is irradiated by a monochromatic pump laser. We will look for squeezing in the fluorescence field in the forward direction. We will treat the cases of resonant excitation (pump frequency $\omega_{p}$ equal to the atomic frequency $\omega_{0}$ ) as well as detuned excitation $\left(\Delta=\omega_{p}-\omega_{0} \neq 0\right)$, of weak exci-

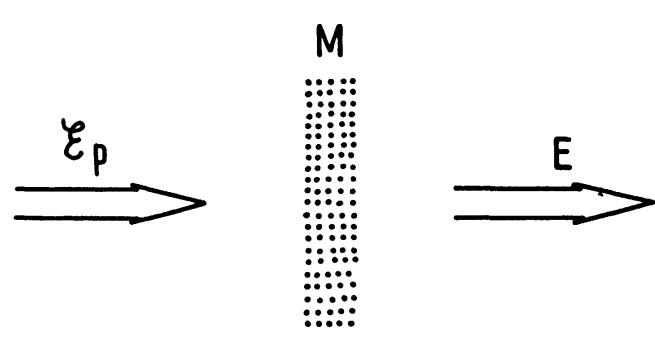

Fig. 1. - The system studied in this paper : the atomic medium $\mathbf{M}$ is irradiated by a pump laser (monochromatic classical field $\varepsilon_{p}$ ). The quantum fluorescence field emitted in the forward direction is denoted $\mathrm{E}$. 
tation (low pump intensity) as well as saturating excitation and finally of homodyne as well as heterodyne detection (see below).

In this paper the study will nevertheless be restricted to a simplified model for the atomic medium. First, atomic motion will be neglected (Doppler effect can be avoided in atomic beam experiments for example). Then we suppose that the two-level atoms are independent and we ignore any collective effect. This corresponds to the approximation of a dilute atomic medium (low density limit). Furthermore we consider only the case of a thin atomic medium where the incident pump laser is not appreciably modified when going through the medium (small optical depth limit).

1.2 ApPlication to PHOTON NOISE REDUCTION. Squeezing may have interesting applications for photon noise reduction [16-18]. Such a photon noise reduction is obtained by entering the squeezed field in one input port of a homodyne or heterodyne device (Fig. 2). The double detector device sketched in figure 2 suppresses excess noise due to local oscillator fluctuations when the power transmission and reflection of the beam splitter are equal [19-20]. Note that the beam splitter of figure 2 can be replaced by a Michelson interferometer adjusted so that its transmission and reflection are equal. The device of figure 2 therefore describes also photon noise reduction in an interferometric device [18].

In a previous paper we have given a quantitative evaluation of the expected reduction factor [21]. We now recall the results which will be used in the following. The signal is the difference $\left(N_{1}-N_{2}\right)$ between the numbers of photons detected on the two detectors

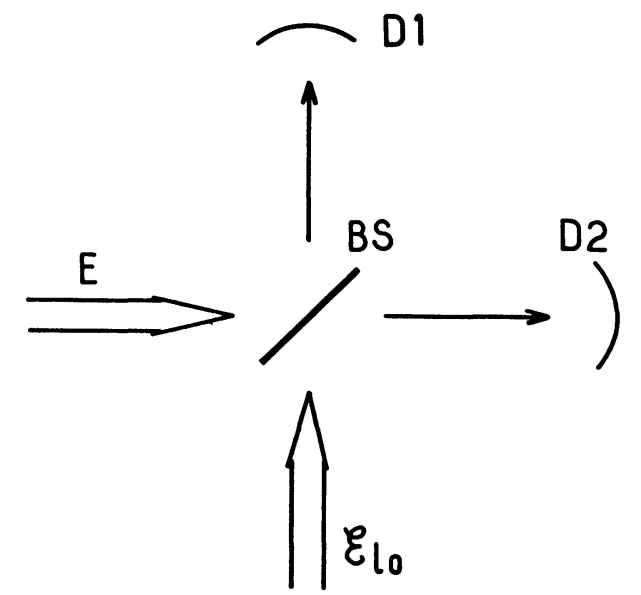

Fig. 2. - Homodyne or heterodyne detection of the squeezed field $E$ with a local oscillator $\varepsilon_{10}$ is achieved by mixing the two fields through a 50-50 beam splitter BS and by detecting the mixed fields on the two photodetectors D1 and D2.

of figure 2 . The variance $\Delta^{2}\left(N_{1}-N_{2}\right)$ of this difference thus measures photon noise. Using photodetection theory [22], one shows that [21] :

$$
\Delta^{2}\left(N_{1}-N_{2}\right)=\bar{N}_{1}+\bar{N}_{2}+C
$$

where the term $C$ describes the deviation from Poisson statistics due to intensity correlations and can be expressed as [21] :

$$
C=C_{0}-C_{2} \cos \psi
$$

with

$$
\begin{gathered}
C_{0}=2 \mathrm{C}^{2} \iiint \int \mathrm{d} t \mathrm{~d} t^{\prime} \mathrm{d}^{2} r \mathrm{~d}^{2} r^{\prime} \varepsilon_{10}^{-}\left(r^{\prime}, t^{\prime}\right) \varepsilon_{10}^{+}(r, t) F^{-+}\left(r, t ; r^{\prime}, t^{\prime}\right) \\
C_{2} \mathrm{e}^{i \psi}=2 \mathrm{C}^{2} \iiint \int \mathrm{d} t \mathrm{~d} t^{\prime} \mathrm{d}^{2} r \mathrm{~d}^{2} r^{\prime} \varepsilon_{10}^{-}\left(r^{\prime}, t^{\prime}\right) \varepsilon_{10}^{-}(r, t) F^{++}\left(r, t ; r^{\prime}, t^{\prime}\right) .
\end{gathered}
$$

The variables $t$ and $t^{\prime}$ run over the detection time $T$, and $r$ and $r^{\prime}$ over the detection area $S$. The local oscillator field is assumed to be in a Glauber coherent state [22] and it is represented by classical fields $\varepsilon_{\mathrm{lo}}^{ \pm}$ $(+$ or - for the positive or negative frequency components). The multiplicative constant $\mathcal{C}$ includes the quantum efficiency $\eta$ of the photodetectors :

$$
\mathcal{C}=\left(2 \varepsilon_{0} c / \hbar \omega\right) \eta .
$$

Expressions (1.2) and (1.3) appear to give the factor $C$ in terms of space time integrals of the correlation functions $\mathrm{F}^{-+}$and $\mathrm{F}^{++}$of the fluctuations $\delta E=$ $E-\langle E\rangle$ of the forward fluorescence field $E$ which enters the device of figure 2 :

$$
\begin{aligned}
& F^{-+}\left(r, t ; r^{\prime}, t^{\prime}\right)=\left\langle\delta E^{-}(r, t) \delta E^{+}\left(r^{\prime}, t^{\prime}\right)\right\rangle \\
& F^{++}\left(r, t ; r^{\prime}, t^{\prime}\right)=\left\langle\delta E^{+}(r, t) \delta E^{+}\left(r^{\prime}, t^{\prime}\right)\right\rangle .
\end{aligned}
$$

The main motivation of this paper is to evaluate these correlation functions and the corresponding photon noise reduction.

1.3 OUTLINE OF THE PAPER. - We first show (§ 2) how the statistical properties of the forward field can be calculated. We then discuss the weak excitation limit where the results are particularly simple $(\S 3)$. We finally include the saturation effects $(\S 4)$ and treat 
non-degenerate heterodyne detection $(\$ 5)$. Our results are compared with previous works in the last section $(\S 6)$.

\section{Calculation of the forward fluorescence field : general framework.}

In this section, we want to show how the statistical properties of the forward fluorescence field can be calculated. As in usual resonance fluorescence theory [23-25], we first relate the fluorescence field to the dipole operators associated with the emitting atoms. We then discuss the expressions obtained for the two correlation functions $\mathrm{F}^{-+}$and $\mathrm{F}^{++}$which describe squeezing.

2.1 RELATION BETWEEN THE FIELD AND THE EMITTING DIPOLES. - The field $E$ transmitted by the atomic medium is obtained as a superposition of the incident pump field $E_{p}$ and of the fields $E_{n}$ radiated by the atoms ( $n=1$ to $N$, where $N$ is the number of atoms) :

$$
E^{+}(r, t)=E_{p}^{+}(r, t)+\sum_{n} E_{n}^{+}(r, t)
$$

(same relation for $E^{-}, E_{p}^{-}, E_{n}^{-}$). Using the fact that the evolution of these dipoles is quasi-monochromatic and supposing that the observation point $r$ is far enough from the emission volume (distance much greater than the wavelength) one gets the simple relation :

$$
E_{n}^{+}(r, t)=\left(K / R_{n}\right) D_{n}^{-}\left(T_{n}\right)
$$

with

$$
\begin{aligned}
& R_{n}=\left|r-r_{n}\right| \\
& T_{n}=t-R_{n} / c
\end{aligned}
$$

where $r_{n}$ is the position of the $n$-th atom and $D_{n}^{-}$the lowering part of the dipole operator associated to this atom :

$$
D_{n}^{-}=d\left|\mathrm{~g}_{n}\right\rangle\left\langle\mathrm{e}_{n}\right|
$$

$\left(\left|g_{n}\right\rangle\right.$ and $\left|e_{n}\right\rangle$ denote the ground and excited states of the $n$-th atom and $d$ the dipole moment). The constant $K$ is equal to :

$$
K=k^{2} /\left(4 \pi \varepsilon_{0}\right)
$$

in the case studied in this paper of forward fluorescence $(k=\omega / c$ wavevector of the pump field).

2.2 Mean Value OF THE FluORESCENCE FiELD. From equations (2.1) and (2.2), one gets the mean fluorescence field :

$$
\left\langle E^{+}(r, t)\right\rangle=\mathcal{E}_{p}^{+}(r, t)+\sum_{n}\left(K / R_{n}\right)\left\langle D_{n}^{-}\left(T_{n}\right)\right\rangle .
$$

The incident field is in a coherent state [22] and we have replaced $\left\langle E_{p}^{+}(r, t)\right\rangle$ by the classical pump field $\varepsilon_{p}^{+}(r, t)$.
Consider first the weak excitation limit. Thus the mean value of the dipole moment is proportional to the incident field :

$$
\left\langle D_{n}^{-}\left(T_{n}\right)\right\rangle=\alpha \mathcal{E}_{p}^{+}\left(r_{n}, T_{n}\right)
$$

where $\alpha$ is the polarizability of the two level atom :

$$
\alpha=-d^{2} /\left(\hbar\left(\Delta+i \frac{\Gamma}{2}\right)\right)
$$

( $\Delta=\omega_{p}-\omega_{0}$ is the detuning and $\Gamma$ the spontaneous emission rate of the excited state).

One deduces :

$$
\left\langle E^{+}(r, t)\right\rangle=\mathcal{E}_{p}^{+}(r, t)+K \alpha \sum_{n} \mathcal{E}_{p}^{+}\left(r_{n}, T_{n}\right) / R_{n}
$$

This expression can be easily evaluated by comparing it to the Huyghens-Fresnel formulation of the propagation law of classical electromagnetics [26]. As a matter of fact, any classical field $\varepsilon$ obeys the integral relation :

$$
\mathcal{E}^{+}(r, t)=(-i / \lambda) \int \mathrm{d}^{2} r_{0} \mathcal{E}^{+}\left(r_{0}, T_{0}\right) / R_{0}
$$

where the point $r_{0}$ is taken on a plane orthogonal to the propagation direction of $\&$ separating the sources of the field and the point of observation (we have used the fact that all the directions of interest are inside a small angle). In equation (2.11), $R_{0}$ and $T_{0}$ are related to $r, t$ and $r_{0}$ in the same manner as in equations (2.3) and (2.4) and $\lambda$ is the wavelength $(\lambda=2 \pi / k)$.

Transforming the discrete summation in (2.10) into an integral :

$$
\sum_{n} f\left(r_{n}\right) \rightarrow \int f(r) \rho \mathrm{d}^{3} r
$$

( $\rho$ volumic density of atoms), and using equation (2.11) for the incident field $\mathcal{E}_{p}$, one gets :

$$
\left\langle E^{+}(r, t)\right\rangle=\varepsilon_{p}^{+}(r, t)[1+i \rho l \lambda K \alpha]
$$

( $l$ is the length of the atomic medium). The field radiated by the atoms is exactly proportional to the incident field and the action of the medium can be described by the usual absorption dispersion theory [27]. Using the expressions of $K$ and $\alpha$ and the relation between $\Gamma$ and $d^{2}$, one gets :

$$
i \lambda K \alpha=-\frac{3 \lambda^{2}}{4 \pi} \Gamma /(\Gamma-2 i \Delta) .
$$

This shows that equation (2.13) gives the usual absorption cross section $\left(3 \lambda^{2} / 2 \pi\right.$ for $\left.\Delta=0\right)$.

Consider now the case in which the atomic transition is saturated by the excitation. The mean dipole can thus be calculated by solving the optical Bloch equations [23]. It is no longer proportional to the field so that the radiated field has not exactly the same 
structure as the incident field. But the HuyghensFresnel principle remains useful for expressing the result. The forward fluorescence field indeed obeys the integral relation (2.11) with a "source» field in the plane of the atomic medium given by :

$$
\mathcal{E}^{+}\left(r_{0}, T_{0}\right)=\mathcal{E}_{p}^{+}\left(r_{0}, T_{0}\right)+i \rho l \lambda K D^{-}\left(r_{0}, T_{0}\right)
$$

where $\mathfrak{D}^{-}\left(r_{0}, T_{0}\right)$ is the mean dipole corresponding to an incident field $\varepsilon_{p}^{+}\left(r_{0}, T_{0}\right)$.

2.3 Correlation FUnCtion $F^{-+}$. - From (2.1), the correlation function $F^{-+}$can be decomposed into :

$$
F^{-+}\left(r, t ; r^{\prime}, t^{\prime}\right)=\sum_{i} \sum_{j}\left\langle\delta E_{i}^{-}(r, t) \delta E_{j}^{+}\left(r^{\prime}, t^{\prime}\right)\right\rangle .
$$

The summation (2.16) includes the incident pump field ( $i=0$ or $j=0$ ) as well as the radiated fields ( $i=1$ to $N$ or $j=1$ to $N$ ). But, as the incident field is in a coherent state, one easily shows that any term corresponding to $i=0$ or $j=0$ is zero. Moreover, the crossed terms $(i \neq j)$ are zero since the fluctuations of two different dipoles are supposed uncorrelated (assumption of a dilute atomic medium). It thus remains :

$$
F^{-+}\left(r, t ; r^{\prime}, t^{\prime}\right)=\sum_{n} K^{2} /\left(R_{n} R_{n}^{\prime}\right) C^{+-}\left(r_{n}, T_{n}, T_{n}^{\prime}\right)
$$

with

$$
C^{+-}\left(r_{n}, T_{n}, T_{n}^{\prime}\right)=\left\langle\delta D_{n}^{+}\left(T_{n}\right) \delta D_{n}^{-}\left(T_{n}^{\prime}\right)\right\rangle
$$

$\left(R_{n}\right.$ and $T_{n}$ (resp. $R_{n}^{\prime}, T_{n}^{\prime}$ ) are related to $r$ and $t$ (resp. $\left.r^{\prime}, t^{\prime}\right)$ through (2.3) and (2.4)). The dipole correlation function $C^{+-}$is well known in resonance fluorescence theory since it is associated to the inelastic part of the fluorescence spectrum [28]. It can be calculated from optical Bloch equations and quantum regression theorem [28]. We will use the corresponding results in the next sections.

The result $(2.17)$ is quite similar to the result of classical coherence theory [29]. In particular, it will be useful in the following to compare it with the Van Cittert-Zernike theorem [26] which describes the propagation of a classical correlation function $\mathcal{F}^{-+}$ (defined as $\boldsymbol{F}^{-+}$in classical electromagnetics). This theorem is easily deduced from the Huyghens-Fresnel principle (2.11) :

$$
\begin{aligned}
& \mathcal{F}^{-+}\left(r, t ; r^{\prime}, t^{\prime}\right)=\left(1 / \lambda^{2}\right) \iint \mathrm{d}^{2} r_{0} \mathrm{~d}^{2} r_{0}^{\prime} \times \\
& \times \mathcal{F}^{-+}\left(r_{0}, T_{0} ; r_{0}^{\prime}, T_{0}^{\prime}\right) /\left(R_{0} R_{0}^{\prime}\right) .
\end{aligned}
$$

The expression (2.17) can be given this form with a correlation function in the plane of sources :

$$
\begin{array}{r}
\mathcal{F}^{-+}\left(r_{0}, T_{0} ; r_{0}^{\prime}, T_{0}^{\prime}\right)=\rho l \lambda^{2} K^{2} \delta^{(2)}\left(r_{0}-r_{0}^{\prime}\right) \times \\
\times C^{+-}\left(r_{0}, T_{0}, T_{0}^{\prime}\right)
\end{array}
$$

(the discrete sum (2.17) has been transformed into an integral). The Dirac function expresses the fact that the sources of field fluctuations are point sources.

2.4 CorRelation FUNCTION $F^{++}$. - The correlation function $\mathrm{F}^{++}$can be decomposed as $\mathrm{F}^{-+}$in equation (2.16). The fact that the incident field is in a coherent state implies that all the terms with $j=0$ are zero but not necessarily the terms with $i=0$. The crossed dipolar terms $(i=1$ to $N, j=1$ to $N$, $i \neq j)$ are still zero so that it remains :

$$
\begin{aligned}
& F^{++}\left(r, t ; r^{\prime}, t^{\prime}\right)= \\
& =\sum_{n}\left\langle\left[\delta E_{p}^{+}(r, t)+\delta E_{n}^{+}(r, t)\right] \delta E_{n}^{+}\left(r^{\prime}, t^{\prime}\right)\right\rangle .
\end{aligned}
$$

It can be shown by careful inspection of the commutation properties between the free incident field and the " source field » operators [15] that equation (2.21) can be transformed into

$$
F^{++}\left(r, t ; r^{\prime}, t^{\prime}\right)=\sum_{n} K^{2} /\left(R_{n} R_{n}^{\prime}\right) C^{--}\left(r_{n}, T_{n}, T_{n}^{\prime}\right)
$$

with

$$
C^{--}\left(r_{n}, T_{n}, T_{n}^{\prime}\right)=\left\langle\mathcal{C}\left[\delta D_{n}^{-}\left(T_{n}\right) \delta D_{n}^{-}\left(T_{n}^{\prime}\right)\right]\right\rangle
$$

where $\mathfrak{C}$ acts as a time ordering operator : it rearranges the order of the two dipole operator so that the former emission time is at the right and the latter one at the left. Time ordering comes out in connection with the fact that the dipole operator commutes with the vacuum fluctuations located in its future but obviously not with those located in its past (causality condition).

The correlation function $C^{--}$is less usual in resonance fluorescence theory than $C^{+-}$but it has also been studied since it describes the squeezing of the field radiated in single atom resonance fluorescence [10-14].

The relation (2.22) is analogous to the relation describing the propagation of a classical correlation function $\mathcal{F}^{++}$(Eq. (2.19) with a multiplicative cons$\operatorname{tant}\left(-1 / \lambda^{2}\right)$ instead of $\left.\left(1 / \lambda^{2}\right)\right)$. The source term is :

$$
\begin{aligned}
\mathcal{F}^{++}\left(r_{0}, T_{0} ; r_{0}^{\prime}, T_{0}^{\prime}\right)=- & \rho l \lambda^{2} K^{2} \delta^{(2)}\left(r_{0}-r_{0}^{\prime}\right) \times \\
& \times C^{--}\left(r_{0}, T_{0}, T_{0}^{\prime}\right) .
\end{aligned}
$$

3. Coherence properties of the forward fluorescence field and photon noise reduction at the weak excitation limit.

We now discuss the weak excitation limit where the results are particularly simple. As we shall see, they have an intuitive interpretation in terms of coherence time and coherence area which will be helpful for discussing more general situations in the next sections. 
3. 1 Dipole CORRELATION FUNCTIONS. - In order to find $F^{-+}$and $F^{++}$, we have first to evaluate the dipole correlation functions $C^{+-}$and $C^{--}$.

The first one, $C^{+-}$, is negligible at the weak excita- tion limit. As a matter of fact, it is associated with the inelastic fluorescence which appears only at the second order in the laser intensity [23].

We now discuss the second correlation function which can be written from equation (2.23) :

$$
\begin{aligned}
C^{--}\left(r_{n}, T_{n}, T_{n}^{\prime}\right) & =\left\langle\delta D_{n}^{-}(T+\tau) \delta D_{n}^{-}(T)\right\rangle \\
& =\left\langle D_{n}^{-}(T+\tau) D_{n}^{-}(T)\right\rangle-\left\langle D_{n}^{-}(T+\tau)\right\rangle\left\langle D_{n}^{-}(T)\right\rangle
\end{aligned}
$$

where $T$ is the former time of $T_{n}$ and $T_{n}^{\prime}$ and $\tau$ the delay between them :

$$
\tau=\left|T_{n}-T_{n}^{\prime}\right| \text {. }
$$

When the delay $\tau$ is larger than the atomic lifetime $1 / \Gamma$, the values of the dipole at $T$ and $T+\tau$ are uncorrelated and $C^{--}$vanishes (the mean value of the product is equal to the product of the mean values). But for $\tau=0$, the first term in equation (3.1), $\left\langle D_{n}^{-}(T) D_{n}^{-}(T)\right\rangle$ is zero (the operator identity $D_{n}^{-} D_{n}^{-}=0$ is easily deduced from Eq. (2.5)) and $C^{--}$is therefore equal to :

$$
-\left\langle D_{n}^{-}(T)\right\rangle^{2}=-\left(\alpha \varepsilon_{p}^{+}\left(r_{n}, T\right)\right)^{2}
$$

where $\alpha$ is the linear polarizability of the atom (see Eq. (2.9)). The transition from $\tau=0$ to $\tau$ infinite can be deduced from the optical Bloch equations through the quantum regression theorem. One finds :

$$
C^{--}\left(r_{n}, T_{n}, T_{n}^{\prime}\right)=-\left(\alpha \varepsilon_{p}^{+}\left(r_{n}, T\right)\right)^{2} \exp \left(-\left(i \omega_{0}+\frac{\Gamma}{2}\right) \tau\right)
$$

(evolution of the dipole at the atomic frequency $\omega_{0}$ and damping with a time constant $2 / \Gamma$ ). Using the definition of $T$ and the fact that $\varepsilon_{p}^{+}$oscillates at the frequency $\omega_{p}=\omega_{0}+\Delta$, one finally gets :

$$
C^{--}\left(r_{n}, T_{n}, T_{n}^{\prime}\right)=-\left(\alpha \varepsilon_{p}^{+}\left(r_{n}, T_{n}\right)\right)\left(\alpha \varepsilon_{p}^{+}\left(r_{n}, T_{n}^{\prime}\right)\right) \exp \left(\left(i \Delta-\frac{\Gamma}{2}\right)\left|T_{n}-T_{n}^{\prime}\right|\right) \text {. }
$$

3.2 COHERENCE PROPERTIES OF THE FORWARD FLUORESCENCE FIELD. - The field correlation function $F^{-+}$ vanishes at the weak excitation limit (since $C^{+-}=0$ ). The second function, $F^{++}$, can be computed from the integral equation (deduced from (2.24)) :

$$
F^{++}\left(r, t ; r^{\prime}, t^{\prime}\right)=\rho l K^{2} \int \mathrm{d}^{2} r_{0} C^{--}\left(r_{0}, T_{0}, T_{0}^{\prime}\right) /\left(R_{0} R_{0}^{\prime}\right)
$$

and from the expression (3.5) of $C^{--}$. This integration is easily performed when using the paraxial approximation (all directions of interest included in a small angle) and the quasimonochromatic evolution (all evolution frequencies included in a narrow band). We suppose that the transverse structure of the incident pump beam is Gaussian with a beam waist $w_{0}$ and no curvature in the plane of the emitting medium. The observation points are supposed to be in the same plane, orthogonal to the propagation direction and located at a distance $R$ from the sources. We will denote $r_{\perp}$ and $r_{\perp}^{\prime}$ the transverse part of the vectors $r$ and $r^{\prime}$. For $R$ large compared to the divergence length of the incident beam (far field observation), one gets :

$$
\begin{aligned}
F^{++}\left(r, t ; r^{\prime}, t^{\prime}\right)=-N \varepsilon_{1}(t) \varepsilon_{1}\left(t^{\prime}\right) \exp [ & \left.i k\left(r_{\perp}^{2}+r_{\perp}^{\prime 2}\right) / 2 R\right] \times \\
& \times \exp \left[-\left(r_{\perp}+r_{\perp}^{\prime}\right)^{2} /\left(2 \phi^{2} R^{2}\right)\right] \exp \left[\left(i \Delta-\frac{\Gamma}{2}\right)\left|t-t^{\prime}\right|\right] .
\end{aligned}
$$

In this equation, $N$ is the number of contributing atom : atoms :

$$
N=\rho l\left(\pi w_{0}^{2} / 2\right)
$$

and $\delta_{1}$ is the mean value of the field radiated by one

$$
\varepsilon_{1}=(K / R) \alpha \varepsilon_{p} \exp (i k R)
$$

( $\xi_{1}$ is calculated at a distance $R$ of the emitting medium and $\varepsilon_{p}$ is the incident field in the plane of this medium; 
both are taken on the axis). The factor $N \varepsilon_{1}^{2}$ which gives the magnitude of the function $F^{++}$could be guessed from the fact that the fluctuations of different dipoles are not correlated. The three exponential functions appearing in equation (3.7) describe the spatial and temporal structure of $F^{++}$and they also have a clear interpretation. The first one means that $F^{++}$has a curvature radius $R$ due to the fact that fluctuations are radiated by point sources at a distance $R$. The second one describes the spatial coherence of the field. Maximum coherence occurs for two points symmetrically located with respect to the axis and the coherence area $\sigma_{\mathrm{c}}$ is given by :

$$
\sigma_{\mathrm{c}}=\phi^{2} R^{2}
$$

where $\phi$ is the angular divergence of the incident pump beam :

$$
\phi=\lambda /\left(\pi w_{0}\right) .
$$

In other words, the coherence solid angle $\Omega_{\mathrm{c}}$ of the fluorescence field is equal to the divergence solid angle of the pump laser :

$$
\Omega_{\mathrm{c}}=\phi^{2} \text {. }
$$

It can also be considered as the diffraction limited solid angle for a source having a surface $\left(\pi w_{0}^{2} / 2\right)$. Finally, the third exponential function in equation (3.7) corresponds to the temporal coherence of the field, directly associated with the temporal correlation of the emitting dipoles (see Eq. (3.5)).

3. 3 Photon NOISE Reduction. - We come now to the evaluation of photon noise which can be expected in a homodyne device (see Fig. 2). The factor $C_{0}$ of equation (1.2) is zero since $F^{-+}=0$. By adjusting the phase between the fluorescence field and the local oscillator, one can thus get a negative value for $C$ :

$$
C=-C_{2} \text {. }
$$

This shows that the fluorescence field emitted by many atoms in the forward direction is squeezed which allows to reduce photon noise $\left(\Delta^{2}\left(N_{1}-N_{2}\right)<\right.$ $\bar{N}_{1}+\bar{N}_{2}$; see Eq. (1.1)).

In order to get an important photon noise reduction, we have to look for conditions where $C_{2}$ is as big as possible. We first choose a detection time $T$ and a detection area $S$ greater than the coherence time $\tau_{\mathrm{c}}$ $(\simeq 1 / \Gamma)$ and coherence area $\sigma_{\mathrm{c}}$ of the fluorescence field (see the discussion in [21]). We have also to match the time and space dependence of the fluorescence field and of the local oscillator.

For discussing this problem, we introduce a new notation $\hat{F}^{++}$through the definition :

$$
\begin{aligned}
F^{++}=\exp [ & \left.-i \omega_{p}\left(t+t^{\prime}\right)\right] \times \\
& \times \exp \left[i k\left(r_{\perp}^{2}+r_{\perp}^{\prime 2}\right) / 2 R\right] \hat{F}^{++} .
\end{aligned}
$$

From equation (3.7) one sees that $\hat{F}^{++}$only depends on the two reduced variables $\tau=\left(t-t^{\prime}\right)$ and $\rho=$ $\left(r_{\perp}+r_{\perp}^{\prime}\right)$. The matching is obtained when the local oscillator frequency (resp. curvature radius) is equal to $\omega_{p}($ resp. $R)$. Under these conditions, the local oscillator field is a spherical wave having its centre on the emitting medium :

$$
\varepsilon_{\mathrm{lo}}^{+}(r, t)=\left|\varepsilon_{\mathrm{lo}}\right| \exp \left[-i \omega_{p} t+i k\left(R+r_{\perp}^{2} / 2 R\right)\right] .
$$

The double time (resp. space) integration in equation (1.3) thus gives the detection time $T$ (resp. detection area $S$ ) on one hand and an integral over $\tau$ (resp. $\rho$ ) on the other hand :

with

$$
C_{2}=\bar{N}_{10} Q_{2}
$$

$$
\begin{gathered}
\bar{N}_{1 \mathrm{o}}=\mathcal{C}\left|\varepsilon_{\mathrm{lo}}\right|^{2} S T \\
Q_{2}=\left|2 \mathcal{C} \iint \mathrm{d} \tau \mathrm{d}^{2} \rho \hat{F}^{++}\right| .
\end{gathered}
$$

The expression (1.1) of the photon noise becomes :

$$
\Delta^{2}\left(N_{1}-N_{2}\right)=\bar{N}_{10}\left(1-Q_{2}\right)+\bar{N}_{\mathrm{f}}
$$

$\left(\bar{N}_{\text {lo }}\right.$ (resp. $\left.\bar{N}_{\mathrm{f}}\right)$ is the number of local oscillator (resp. fluorescence) photons which could be detected; we have used the obvious result $\bar{N}_{1}+\bar{N}_{2}=\bar{N}_{1 \mathrm{o}}+$ $\left.\bar{N}_{\mathrm{f}}\right)$. When the local oscillator field is much more intense than the fluorescence one, the factor $Q_{2}$ appears as the photon noise reduction factor due to the presence of the squeezed field.

The quantitative value of $Q_{2}$ is deduced from equation (3.7) :

$$
Q_{2}=2 \mathrm{CN}\left|\mathcal{E}_{1}\right|^{2}\left(2 \tau_{\mathrm{c}}\right)\left(2 \pi \sigma_{\mathrm{c}}\right)
$$

where $\tau_{c}$ appears as an effective coherence time :

$$
\tau_{\mathrm{c}}=1 /\left|\frac{\Gamma}{2}-i \Delta\right|
$$

and where $\sigma_{\mathrm{c}}$ is the coherence area (see Eq. (3.10)).

3.4 MAGNITUdE OF THE REDUCTION FACTOR. - It is interesting to transform the expression (3.19) of $Q_{2}$ in order to discuss its magnitude. We first note that $\mathcal{C}\left|\varepsilon_{1}\right|^{2} \sigma_{\mathrm{c}}$ is the number of photons emitted by one atom which could be detected on an area $\sigma_{c}$ in a unit time :

$$
\mathcal{C}\left|\varepsilon_{1}\right|^{2} \sigma_{\mathrm{c}}=\Gamma \pi_{\mathrm{e}}\left(\frac{3 \Omega_{\mathrm{c}}}{8 \pi}\right) \eta
$$

where $\Gamma \pi_{\mathrm{e}}$ is the number of photons emitted per atom ( $\pi_{\mathrm{e}}$ population of the excited state for an atom located on the axis), $\eta$ the detection efficiency and ( $3 \Omega_{\mathrm{c}} / 8 \pi$ ) the solid angle factor (which is different from $\Omega_{\mathrm{c}} / 4 \pi$ because the radiation pattern is non spherical). Then, one gets from equations (3.8), (3.11) 
and (3.12):

$$
N \Omega_{\mathrm{c}}=\rho l \lambda^{2} /(2 \pi)
$$

and from equations (3.19), (3.21) and (3.22)

$$
Q_{2}=D_{0}\left(\Gamma \tau_{\mathrm{c}}\right) \pi_{\mathrm{e}} \eta
$$

where $D_{0}$ measures the on resonance optical depth of the atomic medium :

$$
D_{0}=\rho l 3 \lambda^{2} /(2 \pi) .
$$

The reduction factor $Q_{2}$ appears as the product of four dimensionless parameters. It has been calculated in the weak excitation limit and in the case of a thin optical medium. The first assumption implies $\pi_{\mathrm{e}} \ll 1$. The second one requires that the field radiated by the medium is negligible inside the medium itself. From equations (2.13), (2.14) and (3.20), this condition can be written $\left(D_{0} \Gamma \tau_{\mathrm{c}}\right) \ll 1$. As the last factor in equation (3.23), the detection efficiency $\eta$, is smaller than 1 , one obtains a vanishingly small value for the reduction factor.

In the next section, we will release the first assumption by performing a non-perturbative calculation of $Q_{2}$ in the case where the medium remains thin. Releasing the second one is out of the scope of this paper.

\section{Saturation effects.}

When the atomic dipoles are strongly excited, the coherence properties of the field are not so simply described as in the weak excitation limit. The time (resp. spatial) coherence is no longer associated to a mere exponential (resp. Gaussian) variation. However, the photon noise reduction can still be computed. The first step in this calculation is to transform the expressions giving photon noise.

4.1 A USEFUl TRANSFORMATION. - The photon noise is given by expressions (1.1), (1.2) and (1.3) through some integrals over the surface of the detectors. Using the propagation law for the local field oscillator (Huyghens-Fresnel principle (2.11)) and for the fluorescence field correlation functions (Van Cittert-Zernike theorem (2.19)), one easily shows that these integral expressions are conserved when the detection surface is moved along the propagation axis (using the assumption that the detection area is much greater than the coherence area) [15]. It is particularly interesting to bring the integration surface back to the plane of the emitting medium since spatial fluctuations of the field correspond to uncorrelated point sources in this plane (see Eqs. (2.20) and (2.24)). One gets in this manner the expressions (for more detail, see Ref. [15]) :

$$
\begin{aligned}
& C_{0}=2 \mathrm{C}^{2} \lambda^{2} K^{2} \sum_{n} \iint \mathrm{d} t \mathrm{~d} t^{\prime} \varepsilon_{10}^{-}\left(r_{n}, t^{\prime}\right) \varepsilon_{\mathrm{lo}}^{+}\left(r_{m} t\right) C^{+-}\left(r_{m} t, t^{\prime}\right) \\
& C_{2} \mathrm{e}^{i \psi}=2 \mathrm{C}^{2} \lambda^{2} K^{2} \sum_{n} \iint \mathrm{d} t \mathrm{~d} t^{\prime} \varepsilon_{10}^{-}\left(r_{n}, t^{\prime}\right) \varepsilon_{10}^{-}\left(r_{m} t\right) C^{--}\left(r_{w} t, t^{\prime}\right)
\end{aligned}
$$

where $r_{n}$ is the position of the $n$-th atom and where the local oscillator field is virtually defined on the plane of the emitting medium (in fact, $\varepsilon_{10}\left(r_{n}\right)$ is the real field at the point symmetrical to $r_{n}$ with respect to the beam splitter of Fig. 2).

4.2 Evaluation OF THE PHOTON NOISE Reduction. - We now suppose that the local oscillator field evolves at the frequency $\omega_{p}$. Time matching is therefore achieved and the double time integration in equations (4.1) gives the detection time $T$ on one hand and an integral over $\tau=t-t^{\prime}$ on the other hand :

$$
\begin{aligned}
C_{0} & =2 \mathrm{C}^{2} \lambda^{2} K^{2} T \sum_{n}\left|\varepsilon_{10}^{-}\left(r_{n}\right)\right|^{2} \tilde{C}^{+-}\left(r_{n}\right) \\
C_{2} \mathrm{e}^{i \psi} & =2 \mathcal{C}^{2} \lambda^{2} K^{2} T \sum_{n}\left(\varepsilon_{10}^{-}\left(r_{n}\right)\right)^{2} \tilde{C}^{--}\left(r_{n}\right)
\end{aligned}
$$

with

$$
\begin{aligned}
& \tilde{C}^{+-}\left(r_{n}\right)=\int_{-\infty}^{+\infty} \mathrm{d} \tau C^{+-}\left(r_{n}, t, t^{\prime}\right) \exp \left[i \omega_{p}\left(t^{\prime}-t\right)\right] \\
& \tilde{C}^{--}\left(r_{n}\right)=\int_{-\infty}^{+\infty} \mathrm{d} \tau C^{--}\left(r_{n}, t, t^{\prime}\right) \exp \left[i \omega_{p}\left(t^{\prime}+t\right)\right] .
\end{aligned}
$$

The quantities $\tilde{C}^{+-}$and $\tilde{C}^{--}$are nothing but the Fourier transform at frequency $\omega_{p}$ of the dipole correlation functions $C^{+-}$and $C^{--}$. They can be calcu- lated by the methods of single atom resonance fluorescence theory (see for example [23-25] for $\tilde{C}^{+-}$ and $[10-14]$ for $\left.C^{--}\right)$. The results of these calcula- 
tions can be written :

$$
\tilde{C}^{+-}=\left(2 d^{2} / \Gamma\right) A^{+-} ; \quad \tilde{C}^{--}=\left(2 d^{2} / \Gamma\right) A^{--}
$$

with

$$
\begin{gathered}
A^{+-}=p^{+-} / q ; \quad A^{--}=p^{--} / q \\
q=\left(1+2|\varepsilon|^{2}\right)^{3}(1-2 \delta) \\
p^{+-}=4|\varepsilon|^{4}\left[2(1-\delta)^{2}+|\varepsilon|^{2}(1-2 \delta)\right] \\
p^{--}=-2 \varepsilon^{2}\left[(1-\delta)(1-2 \delta)+2|\varepsilon|^{4}\right]
\end{gathered}
$$

where

$$
\begin{gathered}
\left.\varepsilon=-d \varepsilon_{p}^{+}\left(r_{n}\right) / \hbar \hbar\left(\Delta+i \frac{\Gamma}{2}\right)\right] \\
\delta=\Delta /\left(\Delta+i \frac{\Gamma}{2}\right) .
\end{gathered}
$$

The two complex dimensionless parameters $\varepsilon$ and $\delta$ are respectively associated to saturation (the mean dipole moment is just $\mathrm{d} \varepsilon$ at the weak excitation limit; see (2.8) and (2.9)) and to detuning ( $\delta=0$ at resonance).

It now remains to perform the summation over the atoms. Equations (4.2) can be written :

$$
C_{0}=\bar{N}_{1 \mathrm{o}} Q_{0} ; \quad C_{2}=\bar{N}_{\mathrm{lo}} Q_{2}
$$

with

$$
\begin{gathered}
Q_{0}=2 \mathrm{C} \lambda^{2} K^{2} \sum_{n}\left|J\left(r_{n}\right)\right| \tilde{C}^{+-}\left(r_{n}\right) \\
Q_{2}=2 \mathcal{C} \lambda^{2} K^{2}\left|\sum_{n} J\left(r_{n}\right) \tilde{C}^{--}\left(r_{n}\right)\right| \\
\bar{N}_{10}=\operatorname{CT} \int \mathrm{d}^{2} r\left|\varepsilon_{10}^{-}(r)\right|^{2} \\
J(r)=\mathrm{CT}\left(\varepsilon_{10}^{-}(r)\right)^{2} / \bar{N}_{10}
\end{gathered}
$$

and

$\bar{N}_{\text {lo }}$ is the number of local oscillator photons and $|J(r)|$ is the normalized intensity distribution of the local oscillator field.

Two conditions are needed in order to optimize $C_{2}$. First we have to match the curvatures of the fields $\varepsilon_{10}$ and $\varepsilon_{p}$ so that no position dependent phase appears in the term $J\left(r_{n}\right) \tilde{C}^{--}\left(r_{n}\right)$ in equation (4.10). Then the waist of $\varepsilon_{10}$ has to be much smaller than the waist of $\varepsilon_{p}$ in order to avoid a spatial averaging of $\widetilde{C}^{--}$. As in the previous section, this situation corresponds to the case in which the local oscillator field is a spherical wave centred on the emitting plane. One thus gets :

$$
\begin{aligned}
& Q_{0}=2 \mathrm{C} \rho l \lambda^{2} K^{2} \tilde{C}^{+-} \\
& Q_{2}=2 \mathrm{C} \rho l \lambda^{2} K^{2}\left|\tilde{C}^{--}\right|
\end{aligned}
$$

where the correlation functions are calculated for an atom located on the axis. A straightforward transformation finally leads to :

$$
\begin{aligned}
& Q_{0}=D_{0} A^{+-} \eta \\
& Q_{2}=D_{0}\left|A^{--}\right| \eta
\end{aligned}
$$

where $D_{0}$ is the on resonance optical depth (see Eq. (3.24)) and $\eta$ the detection efficiency.

4.3 VARIATION OF THE REDUCTION FACTOR. - When the relative phase between the pump laser and the local oscillator is adjusted, one gets a reduction factor for the photon noise :

$$
\begin{array}{ll} 
& Q=Q_{0}-Q_{2}=D_{0} A \eta \\
\text { with } & A=A^{+-}-\left|A^{--}\right| .
\end{array}
$$

One gets from equations (4.5) and (4.6) :

$$
\begin{aligned}
A^{+-}= & S^{2}[S / 2+2(1-R)(1-S)] \\
\left|A^{--}\right|=S\left[S^{4} / 4+\right. & (1-R)(1-4 R) S^{2}(1-S)^{2}+ \\
& \left.\quad+(1-R)(1-S)^{4}\right]^{1 / 2}
\end{aligned}
$$

where $S$ and $R$ are two real parameters :

$$
\begin{aligned}
& S=2|\varepsilon|^{2} /\left(1+2|\varepsilon|^{2}\right) \\
& R=|\delta|^{2}=\Delta^{2} /\left(\Delta^{2}+\Gamma^{2} / 4\right) .
\end{aligned}
$$

The choice of the « resonance parameter " $R$ between 0 and 1 is equivalent to the choice of the detuning (opposite detunings give the same value of $R$ ). The choice of the "saturation parameter " $S$ between 0 and 1 then determines the intensity of the pump laser.

The variation of $A$ versus $S$ is represented in figure 3 for various values of $R$. First consider the case of exact resonance $(\Delta=0$ and $R=0)$ where $A$ can be

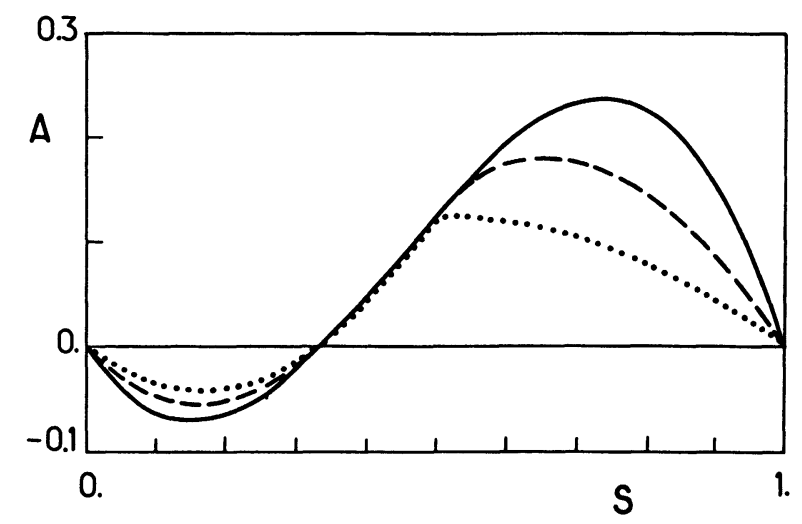

Fig. 3. - The photon noise reduction factor $Q$ is proportional to the parameter $A$ which is represented as a function of the saturation parameter $S$. The solid line corresponds to a resonance parameter $R=0$ (exact resonance), the dashed line to $R=0.5$ and the dotted line to $R=0.75$. 
written :

$$
A=-S(1-3 S)(1-S) .
$$

For small saturation parameters $(S \ll 1), A$ is negative and proportional to $S$ (this is the result of $\S 3$ ). When $S$ increases, the value of $A$ passes through an optimum, then comes back to zero and even becomes positive for $S>1 / 3$. This behaviour is explained by the variations of $A^{+-}$and $\left|A^{--}\right|$ sketched in figure 4. The term $\left|A^{--}\right|$responsible for photon noise reduction increases less rapidly than the saturation parameter $S$. But, the term $A^{+-}$, which is negligible in the weak excitation limit, increases quickly when the transition is saturated and becomes greater than $\left|A^{--}\right|$for $S>1 / 3$.

Curves calculated for excitation out of resonance $(R>0)$ are also represented in figure 3. It clearly appears that the values obtained for $A$ are less interesting than for $R=0$. The solid line of figure 5 represents the variation versus $R$ of the optimum value of $A$ (i.e. the more negative value) obtained when varying $S$. It starts from $(-0.070)$ for $R=0$ and goes to zero when $R$ increases.

Finally, we have shown in this section that the reduction factor for a thin optical medium is proportional to the on-resonance optical depth $D_{0}$, the detection efficiency $\eta$, and a factor $A$ which attains (0.070) in the best conditions $(S \simeq 0.15$ and $R=0$ ).

\section{Non-degenerate heterodyne detection.}

Up to now, we have discussed photon noise reduction in the case where the frequencies of the pump laser and of the local oscillator are equal. We want to introduce an extra degree of freedom by considering the case of a non-degenerate heterodyne detection which can be realized by a spectral analysis at frequency $v$ of the signals delivered by the photodetectors. It can alternatively be realized by modulating at frequency $v$ the local oscillator amplitude (the local oscillator field is thus a bichromatic field with two frequencies $\omega_{p}+v$ and $\omega_{p}-v$ [21]). This extra degree of freedom (choice of $v$ ) will be interesting if the spectrum of the correlation functions responsible for squeezing is important at non-zero frequencies. Note that practical reasons, such as minimization of other noise sources, can also lead to choose heterodyne methods [30-31].

5.1 Evaluation OF THE PHOTON NOISE. - We start from expressions (4.1) which remain valid but we now suppose :

$$
\varepsilon_{10}^{-}\left(r_{n} t\right)=\sqrt{2} \varepsilon_{10}^{-}\left(r_{n}\right) \cos (v t) \exp \left(i \omega_{p} t\right) .
$$

The local oscillator intensity, averaged over a time $T$ much greater than $1 / v$, is $\left|\varepsilon_{10}^{-}\left(r_{n}\right)\right|^{2}$.

In the same manner as in the previous section, one shows that the reduction factor can still be

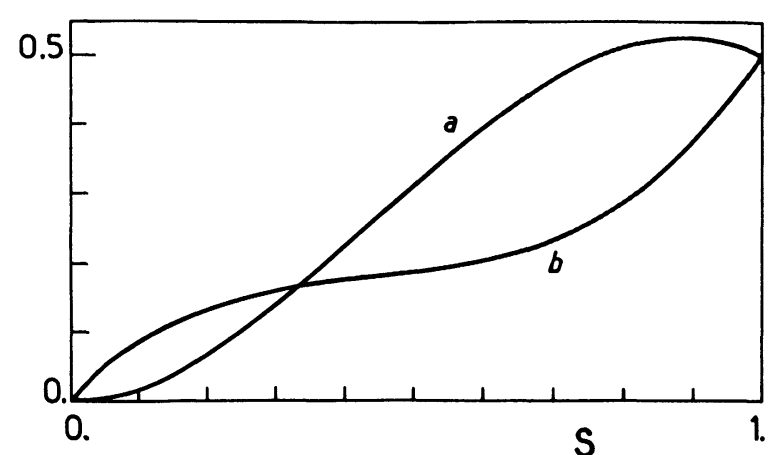

Fig. 4. - The parameter $A$ (see Fig. 3) is the difference between the two terms $A^{+-}$and $\left|A^{--}\right|$which are represented respectively on curves $a$ and $b$ as functions of the saturation parameter $S$, in the exact resonance case $(R=0)$.

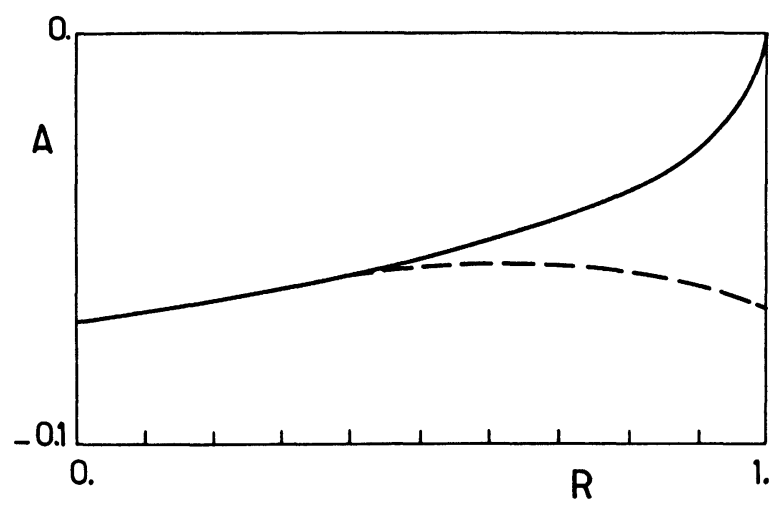

Fig. 5. - The best value of the reduction parameter $A$ attainable by varying the saturation parameter $S$ is plotted as a function of the resonance parameter $R$. Solid line corresponds to the case of homodyne detection (pump laser and local oscillator frequencies equal). Dashed line corresponds to a non degenerate heterodyne detection, where the heterodyning frequency is choosen such that $A$ reaches its best value.

written :

with

$$
Q=D_{0} A \eta
$$

$$
A=A^{+-}-\left|A^{--}\right|
$$

and

$$
A^{+-}=\left(\Gamma / 2 d^{2}\right) \tilde{C}^{+-} ; \quad A^{--}=\left(\Gamma / 2 d^{2}\right) \tilde{C}^{--}
$$

The only difference is that the Fourier transforms $\widetilde{C}^{+-}$ and $\tilde{C}^{--}$of the dipole correlation functions are now taken at frequencies $\omega_{p}+v$ and $\omega_{p}-v$. More precisely, one gets :

$$
\begin{array}{r}
\tilde{C}^{+-}=\int_{-\infty}^{+\infty} \mathrm{d} \tau C^{+-}\left(t, t^{\prime}\right) \exp \left[i \omega_{p}\left(t^{\prime}-t\right)\right] \times \\
\times \cos \left[v\left(t-t^{\prime}\right)\right] \\
\tilde{C}^{--}=\int_{-\infty}^{+\infty} \mathrm{d} \tau C^{--}\left(t, t^{\prime}\right) \\
\exp \left[i \omega_{p}\left(t^{\prime}+t\right)\right] \times \\
\times \cos \left[v\left(t-t^{\prime}\right)\right]
\end{array}
$$


( $\tau=t-t^{\prime}$; compare with Eq. (4.3)). These quantities can still be calculated by the methods of single atom resonance fluorescence theory. One gets :

$$
\begin{aligned}
& A^{+-}=\frac{1}{2}\left(p^{+-}(v) / q(v)+p^{+-}(-v) / q(-v)\right) \\
& A^{--}=\frac{1}{2}\left(p^{--}(v) / q(v)+p^{--}(-v) / q(-v)\right)
\end{aligned}
$$

where $q, p^{+-}$and $p^{--}$are polynomial functions of the dimensionless complex parameters $\varepsilon, \delta$ (defined in Eqs. (4.7), (4.8)) and :

$$
\varphi=v /\left(\Delta+i \frac{\Gamma}{2}\right) \text {. }
$$

One finds :

$$
\begin{aligned}
q(v) & =\left(1+2|\varepsilon|^{2}\right)^{2}\left[(1+\varphi)(1+\varphi-2 \delta)(2+\varphi-2 \delta)+4|\varepsilon|^{2}(1-2 \delta)(1+\varphi-\delta)\right] \\
p^{+-}(v) & =4|\varepsilon|^{4}(1-\delta)\left[(2+\varphi-2 \delta)^{2}+2|\varepsilon|^{2}(1-2 \delta)\right] \\
p^{--}(v) & =-2 \varepsilon^{2}(1-\delta)\left[(1+\varphi-2 \delta)(2+\varphi-2 \delta)-2 \varphi|\varepsilon|^{2}+4|\varepsilon|^{4}\right] .
\end{aligned}
$$

One can now calculate the reduction factor from equations (5.2), (5.3), (5.6) and (5.8) for any values of the pump laser amplitude (parameter $\varepsilon$ ), detuning from resonance $(\delta)$ and heterodyning frequency $(\varphi)$.

5.2 SPECTRAL VARIATION OF THE PHOTON NOISE REDUCTION. - In figure 6 we have plotted the variation of $A$ versus the heterodyning frequency $v$ for various saturation parameters $S$ (defined in (4.18)) in the case of a resonance excitation $(\delta=0$ and so $R=0$; see (4.19)). For a weak excitation (curve a), the best value of $A$ corresponds to $v=0$. For $S=0.15$ (curve b), the value for $v=0$ passes through an extremum (see the discussion in the previous section). For greater saturation parameters (curves $\mathrm{c}$ and $\mathrm{d}$ ), the best value of $A$ is obtained for $v \neq 0$.

The fact that squeezing can be important for nonzero values of $v$ is intimately related to the triplet structure of the fluorescence spectrum $[32,33]$. This can be shown by studying the case of well separated lines $\left(\Gamma \ll \Delta\right.$ or $\left.\mathrm{d} \varepsilon_{p} / \hbar\right)$, i.e. by putting $R=1$ in our formulae. One finds in this limit that $A$ is important only around $v= \pm\left(\Delta^{2}+4\left(\mathrm{dE}_{p} / \hbar\right)^{2}\right)^{1 / 2}$ which is the position of the lateral components of the fluorescence triplet. The variation of $\boldsymbol{A}$ is Lorentzian with the same width as for the triplet. Finally the peak height is :

$$
A=-S(1-S) /[2(1+2 S)] .
$$

It tends to 0 as well for $S \rightarrow 0$ (weak excitation) as for $S \rightarrow 1$ (strong resonance excitation).

5.3 Optimization OF THE REDUCTION FACTOR. We have computed the optimum value reached by $A$ when $v$ and $S$ are varied. The dashed line of figure 5 represents its variation versus $R$.

It clearly appears in figure 5 that there are two ways to get an optimum squeezing. The first one is associated with resonance excitation $(R=0)$ and homodyne detection $(v=0)$. The value $A=-0.070$ can thus be obtained for the optimum saturation parameter $S=0.15$ (see the discussion in the previous section). The alternative choice corresponds to nonresonance excitation $(R=1$ i.e. $\Delta \gg \Gamma)$ and hetero-

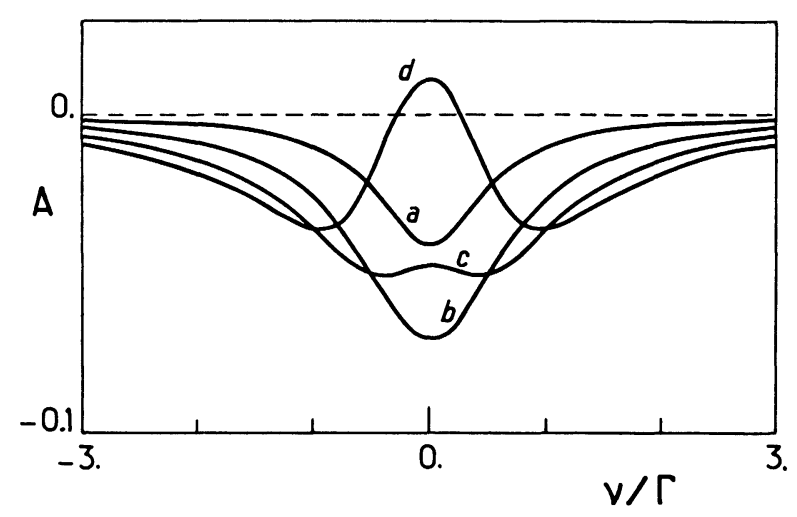

Fig. 6. - Variation of the reduction parameter $A$ versus the heterodyning frequency $v$ (with a frequency unit $\Gamma$ ). Curves $\mathrm{a}, \mathrm{b}, \mathrm{c}$ and $\mathrm{d}$ correspond respectively to a saturation parameter $S$ equal to 0.05 (weak excitation case), 0.15 (the value of $A$ for $v=0$ reaches its best value), 0.25 and 0.35 . All results correspond to a resonant excitation $(R=0)$.

dyne detection at the frequency of the lateral components of the triplet. The value $A=-0.067$ can be obtained for the optimum saturation parameter $S=0.37$.

\section{Conclusion.}

We have shown in this paper that the resonance fluorescence field radiated by a collection of many motionless two level atoms exhibits squeezing. This can seem surprising since non classical statistical properties are usually associated with single atom fluorescence. Nevertheless, theoretical predictions of non classical properties in many-atom resonance fluorescence has already been reported in two papers to our knowledge. For atoms distributed at regular positions, squeezing is expected in the directions of a diffraction pattern [34]. In the much more usual case of randomly distributed atoms, antibunching has been predicted for photodetection points symmetrically located with respect to the propagation axis of the pump laser [35]. These directions are the same as for the squeezing predicted in this paper and the two effects are in fact closely connected. 
We have first studied the perturbative limit and we have given a simple interpretation of the coherence properties of the field in terms of a coherence time (related to the atomic lifetime) and a coherence area (related to the emitting area through the diffraction relation). We have calculated the photon noise reduction which can be expected when the squeezed field is entered in a homodyne detector. As expected from a previous paper [21], the reduction factor is very sensitive to the field coherence properties. It takes the following form through some simple transformations :

$$
Q=D_{0} A \eta
$$

where $\eta$ is the detection efficiency $(0<\eta<1)$, $D_{0}$ the on-resonance optical depth and $A$ a dimensionless factor depending on the correlation functions of the emitting dipoles.

The main result of this paper is equation (6.1) which has been shown to stand also in the cases of saturating excitation and non-degenerate heterodyne detection. The $A$ factor has been calculated for any values of the excitation or detection parameters. It is vanishingly small in the perturbative limit and reaches at best the value $(-0.070)$ for a given value of the saturation parameter.

Equation (6.1) is valid only in the limit of a thin optical medium (small values of $D_{0}$ ). When absorption or dispersion becomes appreciable, the field experienced by the atoms is not only the classical incident field but also the quantum field radiated by the other atoms. This effect can be qualitatively discussed in the case of resonance excitation where $D_{0}$ measures the absorption of the pump beam (no dispersion in this case). One may expect a variation of the reduction factor as follows :

$$
Q \simeq D_{0} \exp \left(-D_{0}\right) A \eta
$$

(same law as in equation (6.1) for $D_{0} \ll 1$ and exponential decay due to absorption for $D_{0} \gg 1$ ). The optimum value of $Q$ would thus correspond to $D_{0} \simeq 1$ :

$$
Q \simeq \exp (-1) A \eta
$$

For the optimum value of $A(A \simeq-0.07)$ and for an ideal quantum efficiency $(\eta=1)$, one would reach in the best conditions :

$$
Q \simeq-0.02
$$

$\left[\Delta\left(N_{1}-N_{2}\right)\right] / \sqrt{\bar{N}_{1}+\bar{N}_{2}}=\sqrt{1+Q} \simeq 0.99$.

This qualitative discussion leads to a reduction which is of the order of $1 \%$ at best.

More elaborate approaches have already been proposed for treating the squeezing produced by a thick optical medium [36-38]. Since they are based on a few mode description of the electromagnetic field, their results can hardly be compared with ours.

Finally, a more satisfactory solution would be to extend the method developed in this paper to thick optical media. This seems feasible although some delicate points have not yet been elucidated.

\section{Acknowledgments.}

We are grateful to C. Cohen-Tannoudji, J. Dalibard, G. Grynberg and A. Aspect for fruitful discussions.

\section{References}

[1] Walls, D. F., Nature 280 (1979) 451.

[2] Loudon, R., Rep. Prog. Phys. 43 (1980) 913.

[3] Schubert, M. and Wilhelmi, B., in Progress in Optics, vol. XVII, ed. E. Wolf (North Holland, Amsterdam) 1980, p. 163.

[4] Dalibard, J. and Reynaud, S., in New Trends in Atomic Physics, ed. G. Grynberg and R. Stora (North Holland) 1984, p. 181.

[5] Kimble, H. J., Dagenais, M. and Mandel, L., Phys. Rev. Lett. 39 (1977) 691.

Dagenais, M. and Mandel, L., Phys. Rev. A 18 (1978) 2217.

[6] Cresser, J. D., Hager, J., Leuchs, G., Rateike, M. and WALTHER, H., in Dissipative Systems in Quantum Optics, ed. R. Bonifacio (SpringerVerlag, Berlin) 1982.

[7] Short, R. and MANDel, L., Phys. Rev. Lett. 51 (1983) 384.

[8] Aspect, A., Roger, G., Reynaud, S., Dalibard, J. and Cohen-TannoudjI, C., Phys. Rev. Lett. 45 (1980) 617.

[9] Walls, D. F., Nature 306 (1983) 141.

[10] Walls, D. F. and Zoller, P., Phys. Rev. Lett. 47 (1981) 709 .

[11] Mandel, L., Phys. Rev. Lett. 49 (1982) 136.

[12] Arnoldus, H. F. and Nienhuis, G., Optica Acta 30 1573 (1983).

[13] Loudon, R., Optics Commun. 49 (1984) 24.

[14] Collett, M. J., Walls, D. F. and Zoller, P., Optics Commun. 52 (1984) 145.

[15] Heidmann, A., Thèse de 3e Cycle (Paris, 1984, unpublished).

[16] Caves, C. M., Phys. Rev. D 23 (1981) 1693.

[17] Quantum Optics, Experimental Gravitation and Measurement Theory, ed. P. Meystre, M. O. Scully (Plenum) 1983.

[18] Reynaud, S. and Heidmann, A., Ann. Phys. Fr. 10 (1985) 227. 
[19] Huen, H. P., Chan, V. W. S., Optics Lett. 8 (1983) 177.

[20] Schumaker, B., Optics Lett. 9 (1984) 189.

[21] Heidmann, A., Reynaud, S. and Cohen-Tannoudi, Optics Commun. 52 (1984) 235.

[22] Glauber, R. J., in Quantum Optics and Electronics, ed. C. de Witt, A. Blandin, C. Cohen-Tannoudji (Gordon and Breach) 1965.

[23] Cohen-Tannoudj, C., in Frontiers in Laser Spectroscopy, ed. R. Balian, S. Haroche and S. Liberman (North Holland) 1977.

[24] Mollow, B. R., in Progress in Optics 19, ed. E. Wolf (North Holland) 1981.

[25] Kimble, H. J. and Mandel, L., Phys. Rev. A 13 (1976) 2123.

[26] Born, M. and Wolf, E., Principles of Optics (Pergamon, 5th edition) 1975.

[27] Feynman, R. P., The Feynman Lectures on Physics, vol. 1 (Addison-Wesley) 1966, ch. 30-7.

[28] Mollow, B. R., Phys. Rev. 188 (1969) 1969.
[29] Mandel, L. and Wolf, E., Rev. Mod. Phys. 37 (1965) 231.

[30] Hall, J. L., Hollberg, L., Baer, T. and Robinson, H. G., Appl. Phys. Lett. 29 (1981) 680.

[31] Bjorklund, G. C. and Levenson, M. D., Phys. Rev. A 24 (1981) 166.

[32] Cohen-Tannoudj,, C. and Reynaud, S., in Multiphoton Processes, ed. J. H. Eberly and P. Lambropoulos (Wiley) 1978, p. 103.

[33] Reynaud, S., Ann. Phys. Fr. 8 (1983) 315.

[34] Vogel, W. and Welsch, D. G., Phys. Rev. Lett. 54 (1985) 1802.

[35] Leberre-Rousseau, M., Ressayre, E. and Tallet, A., Phys. Rev. Lett. 43 (1979) 1314.

[36] Kumar, P. and Shapiro, J. H., Phys. Rev. A 30 (1984) 1568.

[37] ReID, M. D. and Walls, D. F., Optics Commun. 50 (1984) 406.

[38] Kilin, S. Ya., Optics Commun. 53 (1985) 409. 